\title{
Analysing Use Case Diagrams in a Calculus of Context-aware Ambients
}

\author{
Francois Siewe $^{1}$, Ahmed Al-Alshuhai ${ }^{1}$, Saad Almutairi ${ }^{2}$, Abdulgader Almutairi ${ }^{3}$ \\ Software Technology Research Laboratory, De Montfort University ${ }^{1}$, United Kingdom \\ University of Tabuk ${ }^{2}$, Kingdom of Saudi Arabia \\ Al Qassim University ${ }^{3}$, Kingdom of Saudi Arabia
}

\begin{abstract}
Use case diagrams are an excellent tool for capturing and analyzing the functional requirements of a system under development. Context-aware use case diagrams are an extension of use case diagrams to cater for both the functional requirements and the context-awareness requirements of context-aware and pervasive systems. They provide (graphical) notations for specifying, visualizing and documenting the intended behavior of a context-aware system at an early stage of the system development life-cycle. This paper proposes an approach to analyzing contextaware use case diagrams usind a Calculus of Context-aware Ambients (CCA). An algorithm is proposed that translates a context-aware use case diagram into a CCA process. This process can then be analyzed using the CCA tools such as the simulator ccaPL which enables the execution of CCA processes and the model-checker ccaSPIN that can check automatically whether a CCA process satisfies a desired property, e.g. deadlock freedom and reachability. The proposed approach is evaluated using a real-word example of a context-aware pedestrian collision avoidance system.
\end{abstract}

\section{Introduction}

Context-aware computing envisions a new generation of smart systems that have the ability to perpetually sense the user's context and use these data to make adaptation decision in response to changes in the user's context so as to provide timely and personalised services anytime and anywhere. Thanks to the advances in information and communications technology, the emergence of small sensing devices (e.g. GPS, accelerometer, and gyroscope) and miniaturized wireless communication technologies (e.g. blue-tooth, WiFi, and RFID) embedded in small handheld or wearable computing devices such as smartphones is making this paradigm steadily becoming a reality.

Unlike the traditional distribution systems where the network topology is fixed and wired, context- aware computing systems (CASs) are mostly based on wireless communication due to the mobility of the network nodes; hence the network topology is not fixed but changes dynamically in an unpredictable manner as nodes join and leave the network. These factors make the design and development of contextaware computing systems much more challenging as the system requirements change depending on the context of use.

The notion of context-aware use case diagram has been proposed [1] as an abstract, graphical notation for describing the requirements context-aware systems. It is a powerful tool for requirement capturing and analysis at the early stage of the system development life-cycle. More importantly, it seamlessly integrates both the functional requirements and the context-awareness requirements, showing the dependencies between the two types of requirements. However, these use case diagrams can be interpreted manually but are not machine executable. Therefore the analysis of these diagrams may be time consuming and physically demanding, especially for large scale systems. Meanwhile, a machine executable version of these diagrams will ease and speed up requirements analysis a great deal, and enable various scenarios to be tested and validated timely. More importantly, this early system prototype is an effective tool for developers to communicate with the system's end users and domain experts during requirement elicitation and analysis.

The Calculus of Context-aware Ambients (CCA) [2] is a process calculus for modelling context-aware and mobile systems. The main features of the calculus include concurrency, mobility and contextawareness. More importantly, CCA processes are fully executable and can be analysed using the SPIN model-checker [3]

This paper proposes an approach to translating a context-aware use case diagram into a CCA process. This process can then be analysed using the CCA tools such as ccaPL the interpreter and ccaSPIN a model-checking tool based on SPIN. The contribution of this work is threefold: 


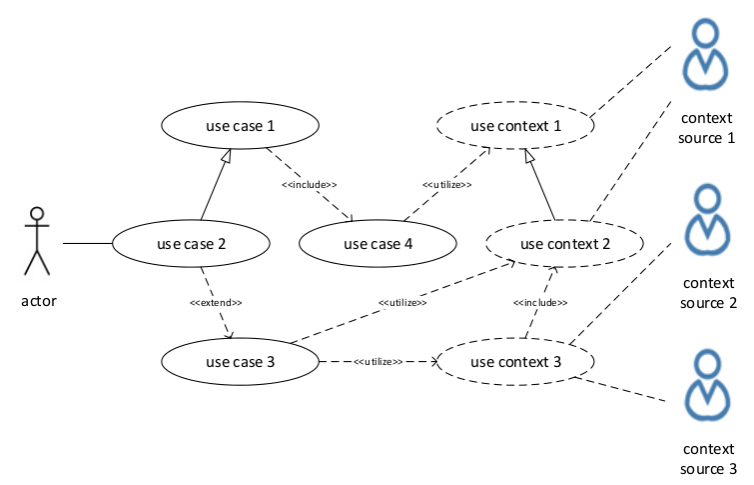

Figure 1. Context-aware use case diagram

- An algorithm is proposed to translate a contextaware use case diagram into a CCA process (Section 4).

- It is demonstrated how ccaPL can be used to analyse system requirements through simulation (Section 5).

- The proposed approach is evaluated using a realword example of a context-aware collision avoidance system (Section 5).

\section{Overview of Context-aware Use Case Diagrams}

A context-aware use case diagram [1] is built from a set of use cases, use contexts, actors, context sources (CSs), and their relationships. Use cases are used to capture the functional requirements of systems. A use case describes the desired behaviour of a system or part of a system (i.e. what a system or part of a system can do), without telling how that behaviour is to be implemented. A use case has a name and is graphically rendered as an ellipse as depicted in Figure 1. Use contexts are used to capture the relevant CIs that affect the behaviour of the system under development, without having to specify how the measurement of those CIs is actually implemented. They also provide the developers a way to come to a common understanding with the system's end user and domain experts as to what CIs the system must be aware of. They are a description of a set of sequence of actions, including variants that a system performs to acquire, to infer or to aggregate CIs from CSs. A use context has a name and is graphically rendered as a dashed ellipse.

An actor represents a coherent set of roles that users of use cases play when interacting with these use cases [4]. Actors can be human or they can be automated systems. An actor is connected to a use case by an association (graphically rendered as a solid line) which indicates that the actor and the use case communicate with one another, possibly by exchanging messages. An actor is represented graphically as a stick figure like in Figure 1. Context sources are to use contexts what actors are to use cases. Use contexts communicate with context sources to gather raw context data from which CIs are calculated. Typically, context sources are sensors; physical sensors (e.g. a temperature sensor or a light sensor) and virtual sensors (e.g. a weather web service or a calendar) alike. Graphically they are rendered as shown in Figure 1. Context sources may be connected to use contexts only by a context association represented by a dashed line.

There are three kinds of relationships between use cases. A generalization relationship between use cases means that the child use case can inherit the behaviour and the meaning of the parent use case; the child may add to or override the behaviour its parent; and the child may be substituted any place the parent occurs [4]. The generalization relationship is represented graphically as a solid directed line with a large open arrowhead. For example in Figure 1, 'use case $1^{\prime}$ is a generalization of 'use case 2'. Conversely, 'use case 2 ' is a specialization of 'use case $1^{\prime}$.

An include relationship between use cases means that the base use case explicitly incorporates the behaviour of another use case; while an extend relationship between use cases means the base use case implicitly incorporates the behaviour of another use case. Graphically, both relationships are rendered as a dependency, stereotyped as <<include〉> and $<<$ extend >> respectively. In Figure 1, 'use case 1' includes 'use case 4' while 'use case 2' extends 'use case 3 '.

These three kinds of relationships also apply to use contexts. An include relationship is used to avoid describing the same CI several times, by putting the common $\mathrm{CI}$ in a use context of its own. An extend relationship is used to model the part of a use context the user may see as optional CI. In this way, optional CIs are separated from mandatory ones. The utilize relationship is the only relationship between a use case and a use context. A utilize relationship between a use case and use context means that the behaviours specified by the use case depend upon the CIs described by the use context. For example, 'use case 3' utilizes 'use context 2' and 'use context 3'. A utilise relationship is graphically rendered as a dependency, stereotyped as <<utilize〉>, like in Figure 1. A utilize relationship always points from a use case towards a use context.

The following section presents the syntax and informal semantics of CCA processes which will be used to model context-aware use case diagrams and analyse them.

\section{Overview of CCA}

The syntax of CCA is depicted in Table 1, based on three syntactic categories: processes (denoted by $\mathrm{P}$ or $\mathrm{Q}$ ), capabilities (denoted by $\mathrm{M}$ ) and contextexpressions (denoted by $\kappa$ ). We assume a countably infinite set of names, elements of which are written in lower-case letters, e.g. $\mathrm{n}, \mathrm{x}$ and $\mathrm{y}$. Keywords are highlighted in bold. 
Table 1. Syntax of CCA

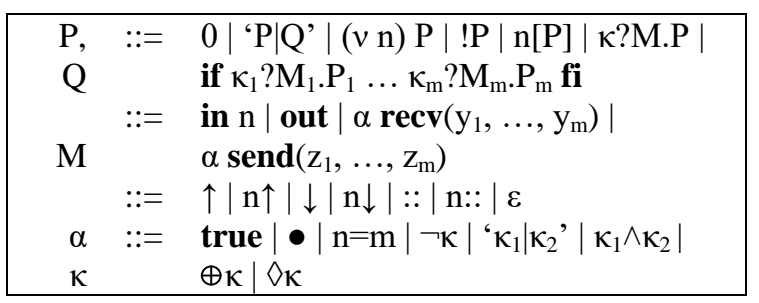

Processes: The process $\mathbf{0}$, aka inactivity process, does nothing and terminates immediately. The process $\mathrm{P} \mid \mathrm{Q}$ denotes the concurrent execution of the process $\mathrm{P}$ and the process $\mathrm{Q}$. The process $(v \mathrm{n}) \mathrm{P}$ creates a new name $n$ and the scope of that name is limited to the process $\mathrm{P}$. The replication !P denotes a process which can always create a new copy of $P$, i.e. !P is equivalent to $\mathrm{P} \mid \mathrm{P}$. Replication, first introduced in the $\pi$-calculus [5], can be used to implement both iteration and recursion. The process $\mathrm{n}[\mathrm{P}]$ denotes an ambient named $\mathrm{n}$ whose behaviours are described by the process $\mathrm{P}$. The pair of square brackets '[' and ']'outlines the boundary of that ambient. An ambient can also be represented graphically as:

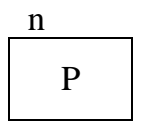

A context expression $\kappa$ specifies a condition upon the state of the environment. A context-guarded prefix $\kappa$ ?M.P is a process that waits until the environment satisfies the context expression $\kappa$, then performs the capability $M$ and continues like the process P. The dot symbol '.' denotes the sequential composition of processes. We let M.P denote the process true?M.P, where true is a context expression satisfied by all context. A selection if $\kappa_{1}$ ? $\mathrm{M}_{1} \cdot \mathrm{P}_{1} \ldots$ $\kappa_{\mathrm{m}}$ ? $\mathrm{M}_{\mathrm{m}} \cdot \mathrm{P}_{\mathrm{m}}$ fi' waits until at least one of the contextexpressions $\left(\kappa_{\mathrm{i}}\right)_{1 \leq \mathrm{i} \leq \mathrm{m}}$ holds; then proceeds nondeterministically like one of the processes $\kappa_{\mathrm{j}}$ ? $\mathrm{M}_{\mathrm{j}} . \mathrm{P}_{\mathrm{j}}$ for which $\kappa_{\mathrm{j}}$ holds and the capability $\mathrm{M}_{\mathrm{j}}$ can be executed.

Capabilities: Ambients exchange messages using an output capability $\alpha \operatorname{send}\left(z_{1}, \ldots, z_{m}\right)$ to send a list of names $\mathrm{z}_{1}, \ldots, \mathrm{z}_{\mathrm{m}}$ to a location $\alpha$, and the input capability $\alpha \operatorname{recv}\left(y_{1}, \ldots, y_{m}\right)$ to receive a list of names from a location $\alpha$ into the variables $\mathrm{y}_{1}, \ldots, \mathrm{y}_{\mathrm{m}}$. The location $\alpha$ can be ' $\uparrow$ ' to mean any parent, ' $n \uparrow$ ' to mean a specific parent $n$, ' $\downarrow$ ' to mean any child ambient, ' $\mathrm{\downarrow}$ ' to mean a specific child $n$, '::' to mean any sibling, ' $\mathrm{n}::$ ' to mean a specific sibling $\mathrm{n}$, or $\varepsilon$ (empty string) to mean the executing ambient itself. The mobility capabilities in and out are defined as follows. An ambient that performs the capability in $\mathrm{n}$ ' moves into the sibling ambient $\mathrm{n}$. The capability out moves the ambient that performs it out of that ambient's parent and into its parent's parent.

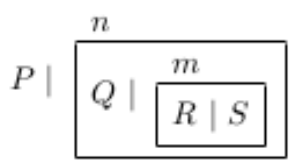

(a) System model

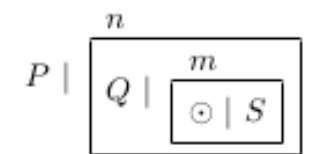

(b) Context of process $R$<smiles>c1ccccc1</smiles>

(c) Context of ambient $m$

Figure 2. Graphical illustration of the context of a process

Table 2. Translating a context-aware use case diagram into a CCA process

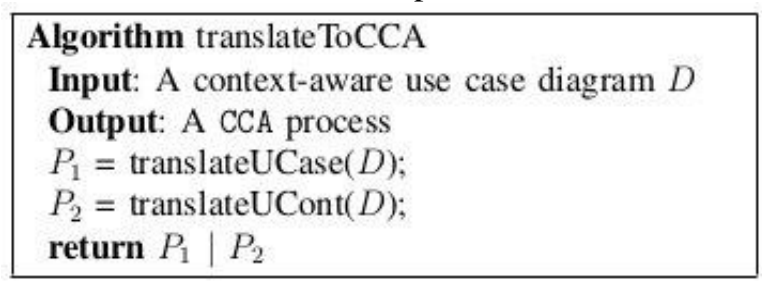

Context model: In CCA, a context is modelled as a process with a hole in it. The hole (denoted by $\odot$ ) in a context is a place holder for the process that context is the context of. For example, suppose a system is modelled by the process ' $\mathrm{P} \mid \mathrm{n}[\mathrm{Q} \mid \mathrm{m}[\mathrm{R} \mid \mathrm{S}]]$ '. The context of the process $\mathrm{R}$ in that system is ' $\mathrm{P} \mid \mathrm{n}[\mathrm{Q} \mid$ $\mathrm{m}[\odot \mid \mathrm{S}]]$ ', and that of the ambient named $\mathrm{m}$ is ' $\mathrm{P} \mid \mathrm{n}[$ $\mathrm{Q} \mid \odot]^{\prime}$ as depicted graphically in Figure 2. A contextexpression is a formula representing some property of a context model.

Context expressions $(C E)$ : The CE true always holds. A CE $n=m$ holds if the names $n$ and $m$ are lexically identical. The $\mathrm{CE} \bullet$ holds solely for the hole context, i.e. the position of the process evaluating that context expression. Propositional operators such as negation $(\neg)$ and conjunction $(\wedge)$ expand their usual semantics to context expressions. A CE $\kappa_{1} \mid \kappa_{2}$ holds for a context if that context is a parallel composition of two contexts such that $\kappa_{1}$ holds for one and $\kappa_{2}$ holds for the other. A CE $n[\kappa]$ holds for a context if that context is an ambient named $n$ such that $\kappa$ holds inside that ambient. A $\mathrm{CE} \oplus \kappa$ holds for a context if that context has a child context for which $\kappa$ holds. A $\mathrm{CE} \nabla_{\kappa}$ holds for a context if there exists somewhere in that context a sub-context for which $\kappa$ holds. The operator $\diamond$ is called somewhere modality, while $\oplus$ is aka spatial next modality.

The following section demonstrates how a context-aware use case diagram can be translated into a CCA process.

\section{Translating Context-aware Use Case Diagrams into CCA Processes}

The algorithm in Table 2 shows how a contextaware use case diagram can be translated into a CCA process. It calls two other algorithms: translateUCase which translates each actor and each 
use case into an ambient (see Table 3); and translateUCont which translates each context source and each use context into an ambient (see Table 4). The final process is the parallel composition of all the ambients so created. Note that associations and dependency relationships are modelled as interactions (i.e. communications) between these ambients.

An actor is modelled as ambient that may interact with any use case it is connected to by sending a message REQUEST_USE_CASE to activate a use case (see (2) in Table 3 ) and receiving notifications as stated in (1). The notation compose $\left(\mathrm{P}_{1}, \ldots, \mathrm{P}_{\mathrm{n}}\right)$ represents one of the four different ways an actor may invoke the use cases it is connected to:

- No invocation: $\operatorname{compose}\left(\mathrm{P}_{1}, \ldots, \mathrm{P}_{\mathrm{n}}\right)=\mathbf{0}$

- Sequentially: $\operatorname{compose}\left(\mathrm{P}_{1}, \ldots, \mathrm{P}_{\mathrm{n}}\right)=\mathrm{P}_{1} \ldots . \mathrm{P}_{\mathrm{n}}$

- Concurrently: $\operatorname{compose}\left(\mathrm{P}_{1}, \ldots, \mathrm{P}_{\mathrm{n}}\right)=\mathrm{P}_{1}|\ldots| \mathrm{P}_{\mathrm{n}}$

- Randomly: compose $\left(\mathrm{M}_{1} \cdot \mathrm{P}_{1}, \ldots, \mathrm{M}_{\mathrm{n}} \cdot \mathrm{P}_{\mathrm{n}}\right)=$ if true? $\mathrm{M}_{1} \cdot \mathrm{P}_{1} \ldots$ true? $\mathrm{M}_{\mathrm{n}} \cdot \mathrm{P}_{\mathrm{n}} \mathbf{f i}$

Any combination of these patterns of actor's behaviours may be considered during simulation and analysis, depending on the system in hand.

Consequently, a use case is modelled as an ambient that receives a request (from one of its actors, or from another use case it extends, or from another use case it is included into) and acquires all the CI it needs by interacting with the use contexts it utilizes and then invokes all the use cases it includes and a subset (possibly empty) of the all the use cases that extend it (see (3) and (4) in Table 3). The function $F_{U}$ in (3) is an abstract representation of the intended behaviours of a use case $\mathrm{U}$; parameterised with that use case interactions with others use cases and use contexts. The concrete specification of this function is system dependent.

A context source is modelled as an ambient that passes fresh sensed raw context values onto use contexts requesting them (see (5) in Table 4). Freshness is modelled by random selection of a value from a representative sample of possible context values. Of course the determination of such sample is system dependent; and hence left to the system designer.

A use context is modelled as an ambient that receives a request from a use case or from another use context that it extends, or from another use context that includes it; then reads all the raw context values it needs from context sources and invokes all the use contexts it includes and a subset (possibly empty) of all the use contexts that extend it. The collected data are used to calculate the CI to be sent to the requester. Similarly to a use case, a use context is an abstraction of what CI a system needs and not how to calculate them. Hence, the actual calculation of the CI is system dependent and therefore cannot be specified in the general case. The function $\mathrm{F}_{\mathrm{C}}$ represents such an abstraction for each use context $\mathrm{C}$.

The CCA process generated by the algorithm in Table 2 can be analysed and animated using CCA tools as shown in the following section.
Table 3. Translating actors and use cases

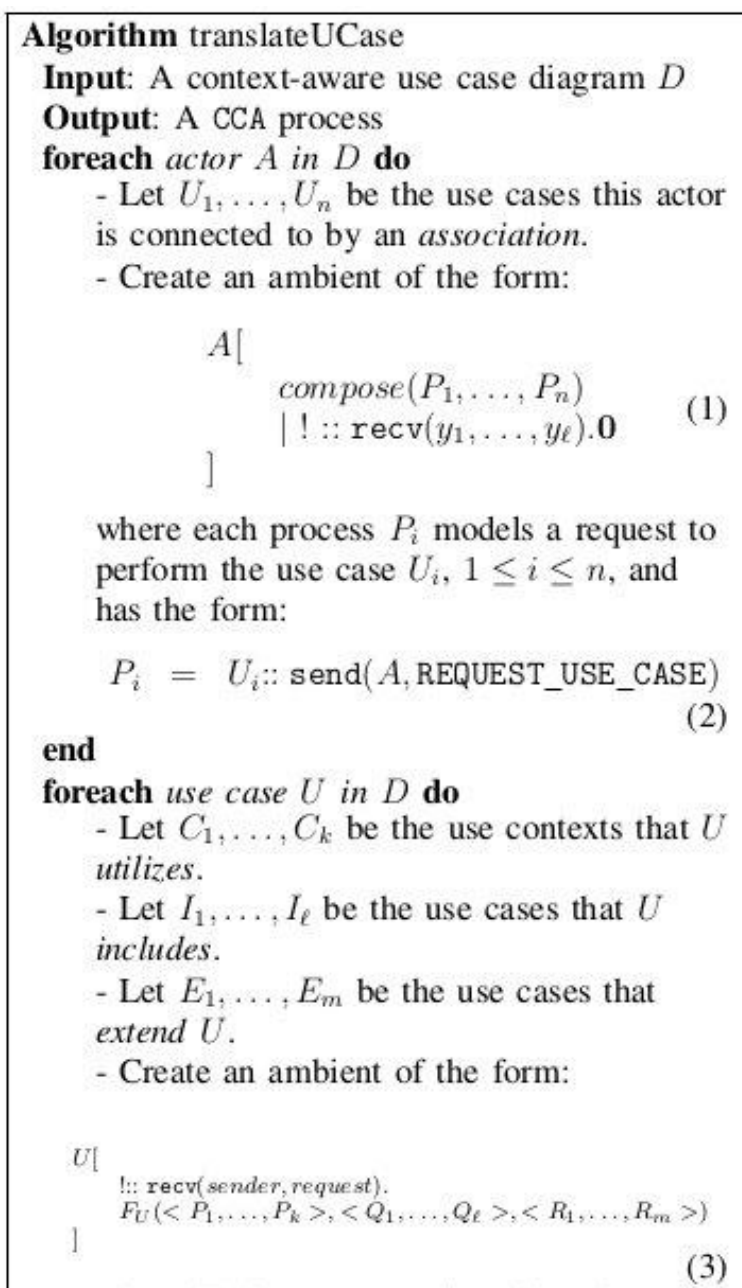

where $F_{U}$ is a process describing the behaviour of the use case $U$ and its parameters are explained as follows: $P_{i}$ is a process modelling the interactions between the use case $U$ and the use context $C_{i}$, $1 \leq i \leq k ; Q_{i}$ is a process modelling the interactions between the use case $U$ and the included use case $I_{i}, 1 \leq i \leq \ell ; R_{i}$ is a process modelling the interactions between the use case $U$ and the extending use case $E_{i}, 1 \leq i \leq m$; they have the forms:

$P_{i}=C_{i}: \operatorname{send}(U, \operatorname{ACQUIRE} C \operatorname{CONTEXT}) \cdot C_{i}:: \operatorname{recv}\left(y_{1}, \ldots, y_{k_{i}}\right)$
$Q_{i}=I_{i}:: \operatorname{send}(U$, CALL_USE_CASE $) \cdot I_{i}:: \operatorname{recv}\left(y_{1}, \ldots, y_{\ell_{i}}\right)$
$R_{i}=E_{i}: \operatorname{send}\left(U\right.$, CALL_USE_CASE). $E_{i}:: \operatorname{recv}\left(y_{1}, \ldots, y_{m_{i}}\right)$

for some non negative integers $k_{i}, \ell_{i}$, and $m_{i}$. The variables sender and request may end occur free in $F_{U}$.

return Parallel composition of all the ambients created. 
Table 4. Translating context sources and use context

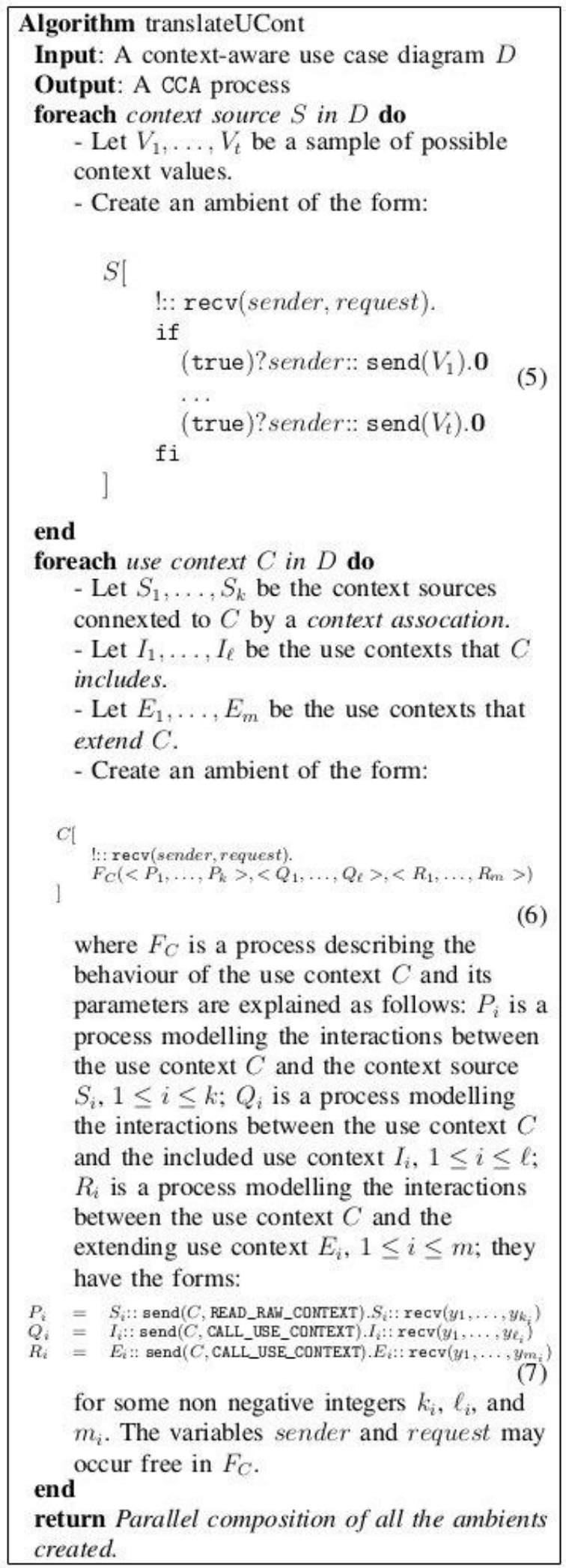

\section{Analysis of Context-aware Use Case Diagrams using CCA}

This section demonstrates how context-aware use case diagrams can be analyzed in CCA using a case study of a pedestrian collision avoidance system. First, a context-aware used case diagram is proposed for the pedestrian collision avoidance system (Section 5.1). Then this context-aware use case diagram is translated into a CCA process using the algorithms presented in Section 4 (Section 5.2). Finally, the resulting CCA process is simulated in ccaPL to understand how the system behaves in a variety of situations (Section 5.3).

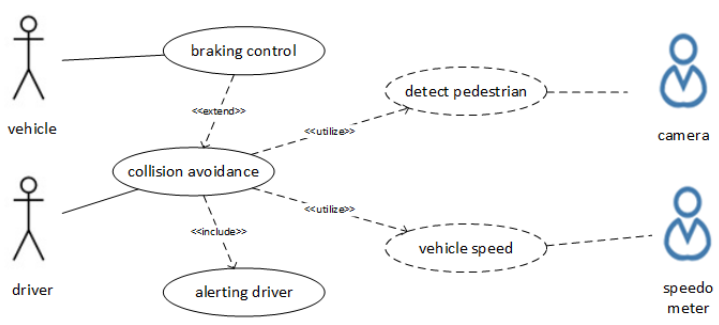

Figure 3. A context-aware use case diagram for a pedestrian collision avoidance system

\subsection{A Context-aware Use Case Diagram for a Pedestrian Collision Avoidance System}

Consider the context-aware use case diagram of Figure 3 for a pedestrian collision avoidance system that enables a vehicle to recognize and respond to potential pedestrian collision situations. The system uses a stereo camera to monitor the path in front of the vehicle and to detect the position and velocity of a pedestrian on the road. A speedometer informs the system of the vehicle current speed. Based on the vehicle speed and the pedestrian position and velocity, the collision avoidance system infers whether a collision may happen in which case the driver is alerted and optionally the braking control is activated. The breaking control applies torque to the wheels to decelerate the vehicle to a safe speed.

There are two actors (driver and vehicle), three use cases (braking control, collision avoidance, and alerting driver), two use contexts (detect pedestrian, and vehicle speed), and two context sources (camera and speedometer). They include relationship between the use case 'collision avoidance' and the use case 'alerting driver' means that the collision avoidance system must always alert the driver of any potential danger of colliding with a pedestrian. However, automatic braking control is optional and this is represented by the extend relationship between the use case 'collision avoidance' and the use case 'braking control'. Moreover, the use case 'collision avoidance' utilizes the use context 'detect pedestrian' to gather CI about the state of the road ahead, e.g. whether there is a pedestrian on the road, the distance of the pedestrian from the vehicle and the pedestrian's velocity. The use context 'vehicle speed' tells how fast the vehicle is moving toward the pedestrian.

\subsection{CCA model of the Pedestrian Collision Avoidance System}

The algorithm translateToCCA depicted in Table 2 can be used to generate a CCA process that models the behaviors of the pedestrian collision avoidance 
system described by the context-aware use case diagram in Figure 3.

The algorithm in Figure 4 says that each actor can be modelled as an ambient. The actor driver can activate the collision avoidance system and receive notifications from the system. This is modelled by the following ambient:

driver[

coll_av::send(driver, REQUEST_USE_CASE)

! ::recv(notification). 0

]

Similarly, the camera senses the position and the velocity of a pedestrian. For the sake of simplicity, the possible values for the position are NONE (no pedestrian detected), CLOSE, and FAR; while the values for the velocity are 0 (zero), SLOW, and FAST. Therefore, the camera can be modelled as an ambient that outputs a randomly pair (position, velocity) representing a possible situation of the pedestrian, i.e.

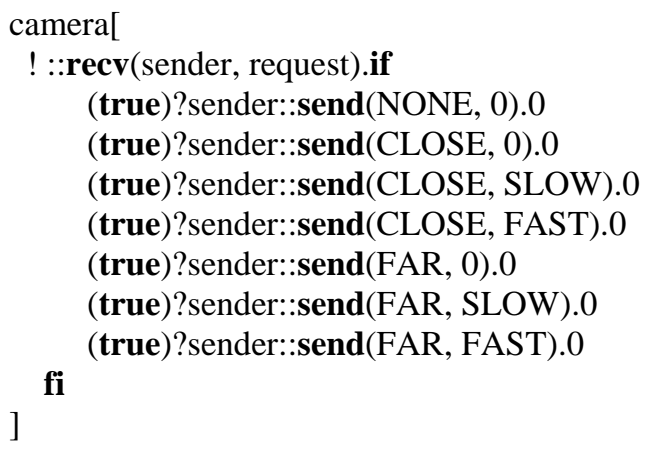

The full CCA process that models the contextaware use case diagram in Figure 3 is depicted in Figure 4, where the ambient coll_av represents the use case 'collision avoidance', the ambient detect_p corresponds to the use context 'detect pedestrian' and the ambient speed models the use context 'vehicle speed'. It is assumed that the speed of the vehicle can be classified as LOW, MEDIUM, or HIGH.

\subsection{Simulation of the Pedestrian Collision Avoidance System}

The CCA process in Figure 4 is randomly simulated in ccaPL and some simulation results are given below. ccaPL represents the simulation output in two forms: textual and graphical. The graphical output (e.g. see Figure 8) resembles the UML sequence diagram showing the involved ambients at the top of the diagram, and the communications between them are depicted as directed arrows from the senders towards the receivers labelled with the message exchanged. The textual simulation output (e.g. see Figure 5) is interpreted as follows. The symbol '-->' represents the reduction relation as defined in the formal semantics of CCA in [2]; it corresponds to one execution step. Each execution step is explained using a notation of the form ' $\{\mathrm{A}$ $===(\mathrm{X})===>\mathrm{B}\}$ ', which means that during that execution step the ambient $\mathrm{A}$ sent the message $\mathrm{X}$ to the ambient B. The following scenarios have been simulated:

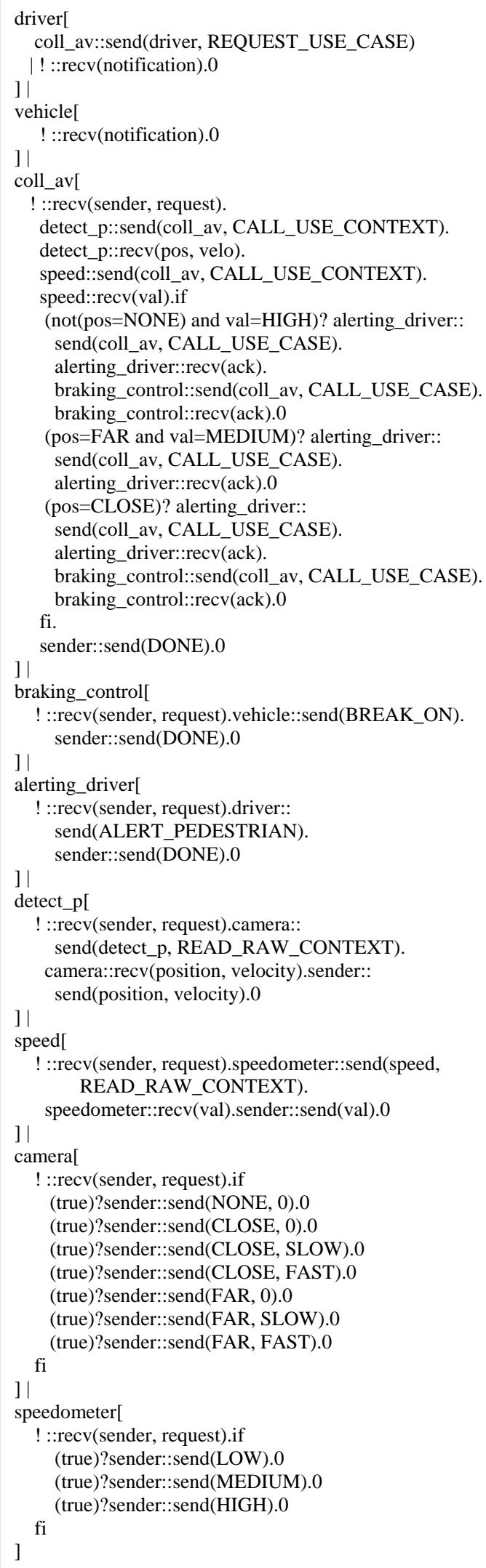

Figure 4. CCA process corresponding to the contextaware use case diagram in Figure 3 
- Scenario 1 (No pedestrian is detected): If no pedestrian is detected then the driver is not alerted and the braking control is not activated as depicted in Figure 5. The corresponding graphical representation is given in Figure 8.

- Scenario 2 (pedestrian is close and vehicle speed is high): If a pedestrian is detected (close and not moving) and the vehicle speed is high then the driver is alerted and the braking control is activated (see Figure 6 and Figure 9).

- Scenario 3 (pedestrian is far away and vehicle speed is low): If a pedestrian is detected and is far away from the vehicle and the vehicle speed is low then the driver is alerted but the braking control is not activated (Figure 7 and Figure 10).

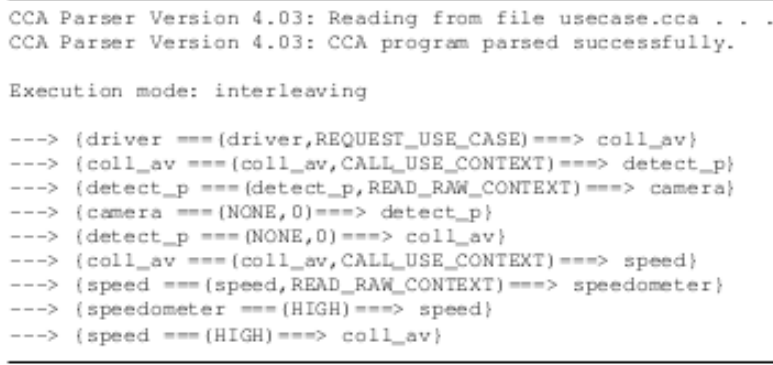

Figure 5. Textual simulation output of scenario 1

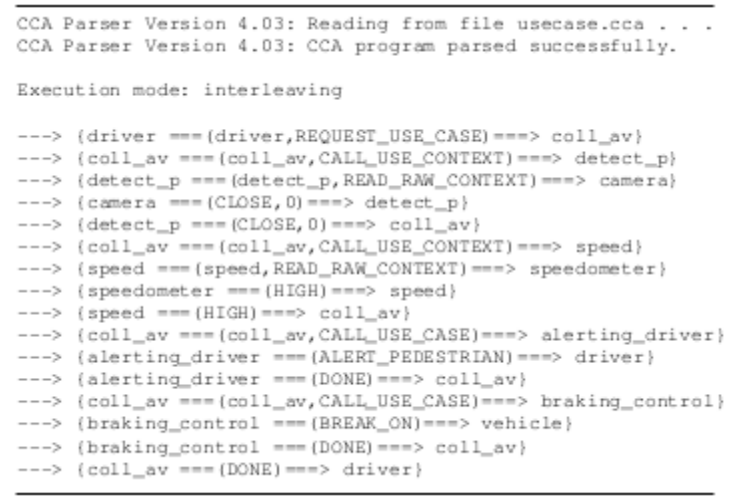

Figure 6. Textual simulation output of scenario 2

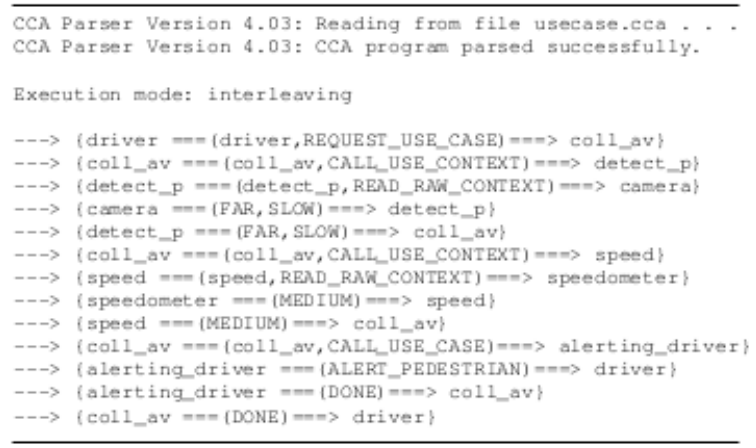

Figure 7. Textual simulation output of scenario 3

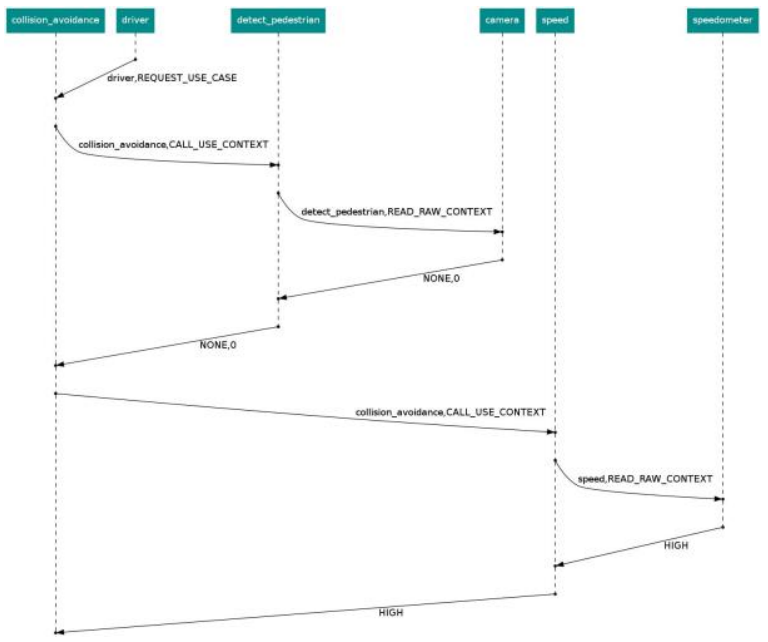

Figure 8. Simulation output of scenario 1

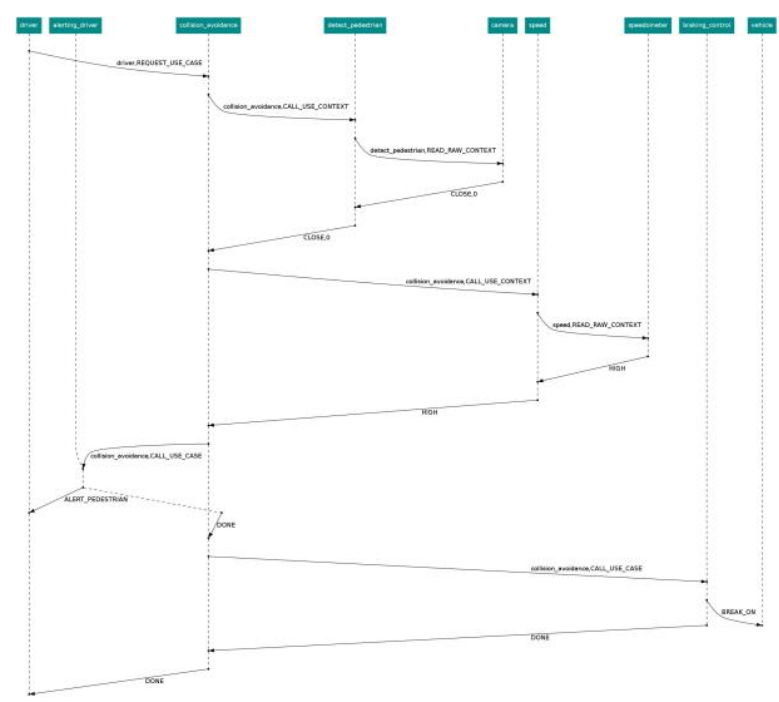

Figure 9. Simulation output of scenario 2

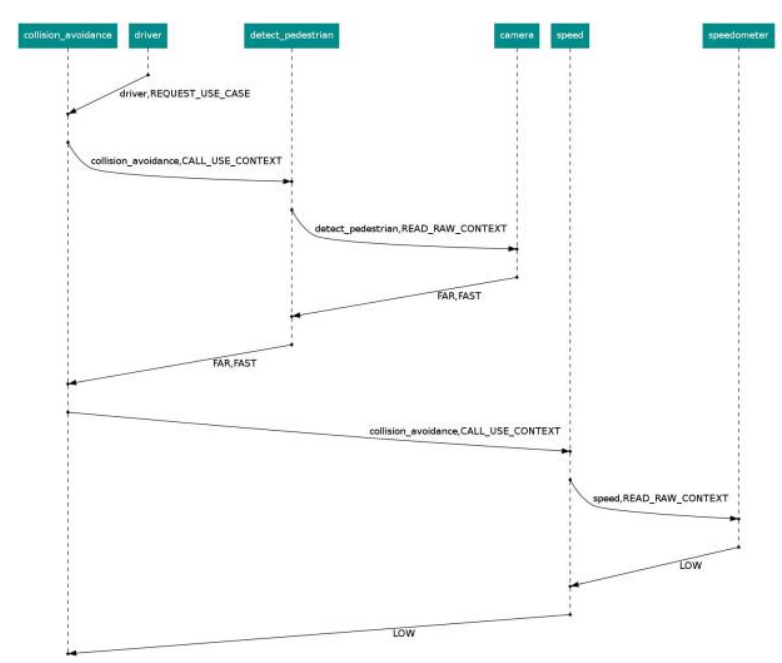

Figure 10. Simulation output of scenario 3

Other scenarios can be defined and simulated in a similar way. 


\section{Related Work}

UML is a diagram language which enables designers of information systems to illustrate high level system requirements, using use case diagrams, and to demonstrate low level system requirements, using activity diagrams [6]. Choi and Lee [7] proposed a model-driven approach that uses UML use case diagrams to elicit the requirement of contextaware systems. In particular, the approach helps analysts and stakeholders pay more attentions to context related issues such as system platform, target users, intelligence, possible context-aware services and agreement with other stakeholders, and understanding contexts with decision tables and trees.

ContUML [8] is a UML-based language for model-driven development of context-aware systems. However, ContUML essentially extends the UML class diagram with special classes for CIs and context-awareness mechanisms. Our context-aware use case diagrams are more abstract than class diagrams and so more suitable for requirement elicitation and analysis. It is understood that ContUML may be used for the realization of contextaware use case diagrams during system development. Almutairi et al. [9] extended the UML use case diagram and activity diagram to capture the security requirements of context-aware system. In particular, they introduces a "requires" relationship between a use case and CIs to indicate the CIs the behaviours described by that use case depend upon. In our approach, use context diagrams are used to specify CIs and their corresponding CSs; separately from the use cases that will utilize those CIs. This separation of concerns between functional requirements and context-awareness requirements is helpful, especially when dealing with large scale or complex contextaware systems.

\section{Conclusion}

This paper proposed an algorithm for translating a context-aware use case diagram into a CCA process in the aim of using the CCA tools to analyse the requirements of context-aware systems. It is demonstrated how the CCA interpreter can be used to execute and validate various scenarios of a use case diagram. The pragmatics of the approach is illustrated using a real-world example of a context-aware pedestrian collision avoidance system. In future work, it will be investigated how the model-checking tool ccaSPIN can be used to analyze the requirements of context-aware systems.

\section{References}

[1] A. Al-Alshuhai and F. Siewe, "An extension of the use case diagram to model context-aware systems," in $S A I$ Intelligent Systems Conference, 2015.

[2] F. Siewe, H. Zedan and A. Cau, "The Calculus of Context-aware Ambients," Journal of Computer and System Sciences, vol. 77, no. 4, pp. 597-620, July 2011.
[3] J. G. Holzmann, "The model checker spin," IEEE Transactions on Software Engineering, vol. 23, no. 5, pp. 117, May 1997.

[4] G. Booch, J. Rumbaugh, and I. Jacobson, The Unified Modeling Language User Guide. Addison Wesley, 1999.

[5] R. Milner, Communication and Mobile Systems: The $\pi$ Calculus. Cambridge University Press, 1999.

[6] A. Finkelstein and A. Savigni, "A framework for requirements engineering for context-awareness services," in First International Workshop from Software Requirements to Architectures, 2001.

[7] J. Choi and Y. Lee, "Use-case driven requirements analysis for context-aware systems," in The Future Generation Information Technology Conference. Springer, 2012.

[8] Q. Z. Sheng and B. Benatallah, "ContextUML: A UMLbased modeling language for model-driven development of context-aware web services," in International Conference on Mobile Business (ICMB05), 2005.

[9] A. Almutairi, A. Abu-Samaha, G. Bella, and F. Chen, "An enhanced use case diagram to model context aware system," in SAI conference, 2013. 